\title{
Screening and diagnosis of gestational diabetes mellitus by IADPSG criteria
}

\author{
Gunjan Chaudhary, Chita Raghunandan, Kanika Chopra*
}

Department of Obstetrics and Gynecology, Lady Hardinge Medical College, New Delhi, Delhi, India

Received: 28 November 2018

Accepted: 29 December 2018

*Correspondence:

Dr. Kanika Chopra,

E-mail: kank2kanu@yahoo.co.in

Copyright: (C) the author(s), publisher and licensee Medip Academy. This is an open-access article distributed under the terms of the Creative Commons Attribution Non-Commercial License, which permits unrestricted non-commercial use, distribution, and reproduction in any medium, provided the original work is properly cited.

\begin{abstract}
Background: GDM is a complex clinical entity and its screening, diagnosis, and management warrants attention. With this issue in mind, we aimed to study the prevalence of gestational diabetes mellitus in the antenatal group of patients attending tertiary hospital in New Delhi and its association with maternal outcome.

Methods: 300 pregnant patients were enrolled in the first trimester and RBS (random blood sugar) was done. Those who had RBS $>200 \mathrm{mg} / \mathrm{dl}$ were labelled as overt diabetes and excluded from the study. The patients who were screened negative in phase 1 of screening were subjected to the next phase of screening at 24-28 weeks of gestation with 2-hour 75g OGTT using IADPSG criteria. Those who were diagnosed as GDM were followed in the antepartum, intrapartum and postpartum period.

Results: The prevalence of overt diabetes and GDM in the study population is $1.33 \%$ and $13.33 \%$. The prevalence of gestational hypertension, pre-eclampsia, chronic hypertension, polyhydramnios was $15 \%, 5 \%, 5 \%, 7.5 \%$ respectively. Normal and instrumental vaginal delivery was in $50 \%, 5 \%$ patients respectively and $45 \%$ had the caesarean section. Conclusions: The single step OGTT with lower threshold value cut-offs and single abnormal value for the diagnosis of GDM as advocated by IADPSG seem to be effective as it helps to screen and diagnose GDM at the same time as a single step procedure and thus more cost-effective.
\end{abstract}

Keywords: Gestational diabetes mellitus, IADPSG criteria, Maternal outcome, Screening

\section{INTRODUCTION}

Diabetes mellitus is defined as disturbance in carbohydrate metabolism characterized by hyperglycemia with peripheral insulin resistance or insulin deficiency. ${ }^{1}$ Diabetes in pregnancy can be of (a) Pre-gestational diabetes/overt diabetes mellitus (b) Gestational diabetes mellitus (GDM). ${ }^{2}$ GDM is carbohydrate intolerance of variable severity with its onset or first recognition during pregnancy. ${ }^{3}$ Risk factors for GDM include advanced maternal age, increased parity, obesity, associated polycystic ovarian syndrome, previous GDM or macrosomic infant and a family history of diabetes. It is a complex clinical entity, with conflicting guidelines and diagnostic protocols. Despite more than 40 years of research, there is no consensus regarding the optimal approach for screening of gestational diabetes. ${ }^{4}$ The global prevalence of diabetes in 2000 was $2.8 \%$ which is likely to increase to $4.4 \%$ in $2030 .^{5}$ In India, the prevalence of GDM ranges from $0.25-5.5 \% .^{6-10}$ There is no uniform international standard to diagnose GDM. Lawrence et al assessed the changes in the prevalence of pre-existing diabetes and GDM from 1999-2005, preexisting diabetes was identified in 2,784 (1.3\%) of all pregnancies, GDM was identified in 15,121 (7.6\%) pregnancies. ${ }^{11}$ Wahi $\mathrm{P}$ et al from Jammu in India recently 
showed the prevalence of GDM as $6.94 \% .^{12}$ In a study conducted in a tertiary care hospital in Haryana Rohtak, the prevalence of GDM was found to be $7.1 \%$ and a single abnormal value was observed in $10.87 \%$ of women. ${ }^{13}$ A study by $\mathrm{K}$ Sreekanthan et al at a medical college in Kollam, Kerala found the prevalence of GDM to be $17 \% .^{14}$ There are evidences now that treating even mild form of GDM is beneficial to the mother and the baby. ${ }^{15}$

Miscarriages and pre-term labour are common in patients with pre-existing diabetes. Antepartum and peripartum complications include pre-eclampsia, polyhydramnios, and operative delivery due to macrosomic baby. The perinatal complications include macrosomia, birth trauma, shoulder dystocia, neonatal hypoglycemia, neonatal respiratory complications, hypocalcemia, polycythemia, stillbirths and increased perinatal mortality. The different diagnostic methods for detecting and diagnosing GDM being employed around the globe today have led several groups to call for the universal adoption and implementation of a single screening and diagnostic strategy. The International collaborative group, recommended a new terminology and a new diagnostic cut offs based primarily on the Hyperglycemia and Pregnancy Outcomes study (HAPO). ${ }^{16}$ Under the new IADPSG (International Association of Diabetes and Pregnancy Study Groups), there is a two-phase strategy of screening all pregnant women for diabetes mellitus at first antenatal visit in first trimester and with one step $75 \mathrm{~g}$ OGTT at 24-28 weeks of pregnancy.

This proposed new strategy would include changing from a 2-step to a 1- step screening process, along with a lower glucose threshold for the diagnosis of GDM. Universal screening for GDM detects more cases and improves maternal and offspring prognosis compared to selective screening. ${ }^{17}$ Present study was thus aimed to determine the prevalence of gestational diabetes mellitus in the antenatal group of patients registered in our hospital and to study the association of gestational diabetes mellitus with maternal outcome.

\section{METHODS}

Present study was an observational study conducted from November 2013 to March 2015 in antenatal outpatient department and labour room of Lady Hardinge Medical College, New Delhi. Sample size was calculated using the formula $\mathrm{N}=4 \mathrm{PQ} / \mathrm{L} 2$ where $\mathrm{P}$ is 10 , $\mathrm{Q}$ is $100-\mathrm{P}$ and $\mathrm{L}$ is 4 i.e. allowable error. It calculated as 225 and so we rounded the figure as 300 . So, a total of 300 pregnant women registered at the antenatal clinic with single live fetus in first trimester were enrolled randomly in the study after taking informed consent.

\section{Exclusion criteria}

- Patients with history of renal, liver, thyroid disorder and on medications such as steroids or thiazides.
In phase 1 of screening random blood sugars of all pregnant women were measured. Samples were analyzed on fully automated clinical chemistry analyzer AU480 (Olympus, Beckman coulter, USA), using commercially available kit provided by Randox, UK using GOD-POD method. Random blood sugars > $200 \mathrm{mg} / \mathrm{dl}$ were labelled as overt diabetes and treated accordingly. Those who were tested negative i.e. random blood sugars $<200$ $\mathrm{mg} / \mathrm{dl}$ were included in phase 2 of screening and at 24-28 weeks of gestation, 2-hour $75 \mathrm{gm}$ OGTT was done for them. IADPSG criteria was followed in which fasting blood sugars $\geq 92 \mathrm{mg} / \mathrm{dl}, 1$ hour $\geq 180 \mathrm{mg} / \mathrm{dl}$ and 2 hours $\geq 153 \mathrm{mg} / \mathrm{dl}$ were considered abnormal. Overt diabetes was defined as fasting plasma glucose levels $>126 \mathrm{mg} / \mathrm{dl}$, GDM as one or more values exceeding IADPSG thresholds and normal as all 3 glucose values less than threshold.

Those who were tested positive as per the IADPSG criteria were managed with dietary modification, lifestyle modification, exercise regimen, insulin /hypoglycemic agents as per the hospital protocol taking target therapy value as fasting blood glucose levels less than $95 \mathrm{mg} / \mathrm{dl}$ and two-hour postprandial blood glucose levels less than $120 \mathrm{mg} / \mathrm{dl}$. The women having GDM were kept under intense surveillance by SMBG (self-monitoring of blood glucose) and were screened for complications like preeclampsia and polyhydramnios.

Ultra-sonography, for fetal biometry and amniotic fluid indices was carried to monitor fetal growth, three weekly USG was done to detect macrosomia during the antenatal checkups. Antepartum fetal surveillance was done by daily fetal movement count, non-stress test, and biophysical profile score.

The time and mode of delivery was individualized. Some of the patients went in spontaneous labour and were directly admitted to the labour room and some of them were admitted in wards and were induced and some of them were electively taken up for cesarean due to good size baby.

The patients were followed up in the labour room for the intrapartum events till the delivery of the baby. The patient's blood sugar monitoring was strictly done in the first, second and the third stage of labor to maintain good glycemic control ( 2 hourly dextrose charting and 4 hourly random blood glucose) while avoiding hypoglycemia.

The birth weight of babies was estimated using the weighing machine and baby was examined by pediatrician to rule out any birth injury. So, the outcome of present study was to detect the prevalence of Gestational Diabetes Mellitus in pregnant women following universal screening and one step OGTT and to study the association of GDM with adverse maternal complications of pre-eclampsia and polyhydramnios and its association with caesarean delivery for CPD, shoulder dystocia and birth trauma. 


\section{Statistical analysis}

Statistical tests were performed using the available version of SPSS software and values of $\mathrm{p}<0.05$ were considered significant. Mean and standard deviation will be reported for continuous variable and no and \% will be reported for categorical variables. Present study was approved by the ethical committee of our institute.

\section{RESULTS}

In present study, 300 patients were recruited for Phase 1 of screening. 4 patients were excluded from the study after being diagnosed as overt diabetes. The prevalence of overt diabetes in present study population was $1.33 \%$. The remaining 296 were screened with 2-hour $75 \mathrm{~g}$ OGTT at 24-28 weeks as per IADPSG criteria in Phase 2 of screening. 40 patients out of 296 were tested positive for GDM which constituted the study group. The prevalence of gestational diabetes mellitus following one step OGTT was $13.33 \%$.

The demographic and clinical parameters of the study group is shown in Table 1.

Table 1: Demographic characteristics and clinical parameters of study group (GDM positive patients).

\begin{tabular}{|l|l|}
\hline$(\mathrm{N}=40)$ & Study group \\
\hline Mean age \pm SD $($ years $)$ & $28.75 \pm 4.73$ \\
\hline Mean gravidity \pm SD & $2.5 \pm 0.574$ \\
\hline Mean height \pm SD $(\mathrm{cm})$ & $156 \pm 4.64$ \\
\hline Mean weight \pm SD $(\mathrm{kg})$ & $70.90 \pm 9.58$ \\
\hline Mean BMI \pm SD $\left(\mathrm{kg} / \mathrm{m}^{2}\right)$ & $29.15 \pm 4.96$ \\
\hline Mean SBP \pm SD $(\mathrm{mm} \mathrm{hg})$ & $126.28 \pm 12.29$ \\
\hline Mean DBP \pm SD $(\mathrm{mm} \mathrm{hg)}$ & $79.85 \pm 1.01$ \\
\hline
\end{tabular}

Table 2 shows distribution of study group on the basis of past history in study group. The mean RBS value in study population was $96.64 \pm 25.74$ whereas in study group it was $109.32 \mathrm{mg} / \mathrm{dl}$. The mean fasting, $1 \mathrm{sthr}, 2 \mathrm{ndhr}$ in the study population were $76.5 \pm 16.26,125.57 \pm 33.16$, $111.64 \pm 28.99$ respectively.

Table 2: Distribution of study group on the basis of past history.

\begin{tabular}{|l|l|l|}
\hline History & Frequency & $\%$ \\
\hline Family history of DM & 6 & 15 \\
\hline Previous abortion & 9 & 22.5 \\
\hline Preterm deliveries & 1 & 2.5 \\
\hline Macrosomia & 2 & 5 \\
\hline Still birth & 3 & 7.5 \\
\hline Shoulder dystocia & 1 & 2.5 \\
\hline
\end{tabular}

Out of abnormal thresholds of fasting, $1^{\text {st }}$ hour and $2^{\text {nd }}$ hour values, the mean fasting value was $98.64 \mathrm{mg} / \mathrm{dl}$, the mean $1 \mathrm{st}$ hour value was $197.117 \mathrm{mg} / \mathrm{dl}$ and the mean $2^{\text {nd }}$ hour value was $167.12 \mathrm{mg} / \mathrm{dl}$ as seen in Table 3 .
Table 3: Mean blood sugar values in total study population and group.

\begin{tabular}{|l|l|l|}
\hline RBS & $\begin{array}{l}\text { Total study population } \\
(\mathbf{n = 3 0 0})\end{array}$ & $\begin{array}{l}\text { Study group } \\
(\mathbf{n = 4 0 )}\end{array}$ \\
\hline Fasting & $96.64 \pm 25.74$ & $109.32 \pm 28.02$ \\
\hline 1 hour & $16.5 \pm 16.26$ & $98.64 \pm 5.54$ \\
\hline 2 hours & $111.64 \pm 28.99$ & $197.117 \pm 20.11$ \\
\hline
\end{tabular}

In the total of 40 patients, 20 had only one value deranged, 16 had two values deranged and 4 had all three values deranged. Among the women who had only one value deranged, only fasting value was deranged in 11 , only $1^{\text {st }}$ hour value was deranged in 4 and only $2^{\text {nd }}$ hour value was deranged in 5 (Table 4).

Table 4: Frequency of single abnormal blood sugar value in study group.

\begin{tabular}{|l|l|r|r|}
$\mathrm{N}=20$ & Fasting & 1 hour & 2 hours \\
\cline { 2 - 4 } & $11 \mathrm{mg} / \mathrm{dl}$ & $4 \mathrm{mg} / \mathrm{dl}$ & $5 \mathrm{mg} / \mathrm{dl}$ \\
\hline
\end{tabular}

These 40 women diagnosed with GDM were followed up till delivery for the antenatal, intrapartum and postpartum events. Out of 40, 30 patients had normal BP records, i.e. BP $<140 / 90 \mathrm{~mm} \mathrm{Hg} .8$ patients had Pregnancy Induced Hypertension (PIH) in which 2 patients had pre-eclampsia i.e. BP $>140 / 90 \mathrm{~mm} \mathrm{Hg}$ associated with proteinuria and 6 patients had BP $>140 / 90$ $\mathrm{mm} \mathrm{Hg}$ without proteinuria and 2 patients had chronic hypertension. There were no cases with eclampsia. Hence, the prevalence of gestational hypertension in study group was $15 \%$, of pre-eclampsia was $5 \%$ and of chronic hypertension was 5\%. 3 patients out of 40 patients had polyhydramnios contributing to $7.5 \%$ of the study group. 20 out of $40(50 \%)$ of patients had normal vaginal delivery, 2 out of $40(5 \%)$ of patients had instrumental delivery ( 1 forceps and 1 vacuum) and 18 out of $40(45 \%)$ patients had caesarean section. Only 1 $(2.5 \%)$ baby had birth injury and birth asphyxia was observed in 2 out of 40 patients $(5 \%)$.

\section{DISCUSSION}

The prevalence of gestational diabetes mellitus is on the rising trend and is known as one of the most common complications during pregnancy due to increase in the number of women of obesity among the women of child bearing age. ${ }^{18}$ Early detection and management of gestational diabetes mellitus improves the outcomes for both the mother and the fetus that includes treatment of even mild GDM. ${ }^{15}$ HAPO study, hyperglycemia and adverse pregnancy outcome study reported that glucose levels lower than that was required for the diagnosis of gestational diabetes mellitus were significantly associated with adverse pregnancy outcomes. ${ }^{16}$ IADPSG criteria was similarly based on HAPO study, and thus recommended new diagnostic criteria for the diagnosis of GDM. ${ }^{19,20}$ IADPSG criteria has found a fairly wide acceptance since 
the time of its introduction. As per the IADPSG criteria, GDM was diagnosed using fasting blood sugar levels in the first trimester and oral glucose tolerance test in 2nd/3rd trimester.

Screening in the first trimester helps in detecting overt diabetes and also universal screening at 24-28 weeks is more sensitive than selective screening recommended by American Diabetic Association. ${ }^{21}$ So, we planned this study to determine the prevalence of gestational diabetes mellitus and to study its association with maternal outcomes i.e. hypertension, polyhydramnios and caesarean delivery.

Table 5: Studies on prevalence of GDM.

\begin{tabular}{|l|l|}
\hline Studies & Prevalence of GDM \\
\hline Bhavadharini et $\mathrm{al}^{22}$ & 18.5 \\
\hline $\mathrm{O}^{\prime}$ Sullivan et $\mathrm{al}^{23}$ & 12.4 \\
\hline Gopalakrishnan V et $\mathrm{al}^{24}$ & 41.9 \\
\hline Hung et $\mathrm{al}^{25}$ & 12.4 \\
\hline Arora et al $^{26}$ & 34.9 \\
\hline${\text { Nayak et } \mathrm{al}^{27}}^{\text {Dahanayaka et } \mathrm{al}^{28}}$ & 27 \\
\hline
\end{tabular}

The prevalence of overt diabetes and GDM in present study population was $1.33 \%$ and $13.33 \%$ respectively. The prevalence of gestational diabetes mellitus has been studied worldwide using IADPSG criteria as shown in table 522-28. In majority of studies the prevalence of GDM was comparable to present study. Higher prevalence rate reported in a study by Arora et al, was due to larger sample size and they used capillary blood sample rather than venous sample as in present study. In a study done by Bhavadhari et al, screening for GDM started in first trimester and then between 24-28 weeks of gestation as in present study, thus detecting prevalence of overt diabetes as $1.2 \% .{ }^{22}$ Fasting hyperglycaemia was seen in $55 \%$ of our patients in present study. Gopalakrishnan et al reported that $70.5 \%$ patients had fasting hyperglycemia and in a study by Moradi et al, $48 \%$ of GDM diagnosed using IADPSG criteria had elevated fasting blood sugar levels alone. ${ }^{24,29}$

In a study by $M$ Shang et al, an IADPSG criteria was used to detect the prevalence of gestational diabetes mellitus and predicting adverse outcomes. ${ }^{30}$ The prevalence of GDM in this study was $19.9 \%$. The prevalence of preeclampsia in GDM patients was $7.2 \%$ and caesarean section was done for $54.9 \%$ patients. In this study the prevalence of GDM was calculated using both IADPSG and ADA criteria and also prevalence of adverse pregnancy outcomes was compared between patients with gestational diabetes mellitus and those with normal glucose tolerance. Present study on the other hand, did not include the controls with normal blood sugar values. If it would have been included results would have been clearer and statistically significant. Further studies are necessary to replicate the benefits of correctly diagnosing and managing GDM in larger populations.

\section{CONCLUSION}

The single step OGTT with lower threshold value cut offs and single abnormal value for diagnosis of GDM as advocated by IADPSG seems to be effective because of its association with adverse pregnancy outcomes. It is anticipated that lowering the diagnostic criteria for GDM will lead to early diagnosis and effective management of patients with hyperglycemia starting from first trimester of pregnancy as well. Due to relatively small number of patients in present study, further studies with large sample size are required to ascertain the increased prevalence of GDM using IADPSG criteria.

\section{Funding: No funding sources \\ Conflict of interest: None declared \\ Ethical approval: The study was approved by the Institutional Ethics Committee}

\section{REFERENCES}

1. American Diabetes Association Diabetes Care. 2011;34(1):S62-9.

2. International Diabetes Federation. Diabetes Atlas 2011, $5^{\text {th }}$ ed. Brussels, Belgium: IDF 2012.

3. Cunningham FG, Lenovo KJ, Bloom SL, Haut JC, Rouse DJ, Spongy CY. Diabetes. In: Williams Obstetrics. New York: MC Graw-Hill, 2010; pp. 1104-1125.

4. ACOG Practice Bulletin. Gestational Diabetes 2001;98(3);525-37.

5. Wild S, Roglic G, Green A, Sicree R, King H. Global prevalence of diabetes: estimates for the year 2000 and projections for 2030. Diabetes Care. 2004;27(5):1047-53.

6. Bhattacharya C, Awasathi RT, Kumar S, Lamba PS. Routine screening for gestational diabetes mellitus with glucose challenge test in antenatal patients. J Obstet Gynaecol India. 2001;51:75.

7. Ganguly, A. A Study of Diabetes Mellitus over 8 years. J Obstet Gynecol India.1995;45:27-31.

8. Goel N, Bathla S. Diabetes in pregnancy. Obstet Gynaecol Today 1999;46:135.

9. Kumar A, Takkar D, Sunesh K. Diabetes complicating pregnancy. J Obstet Gynaecol India 1993;43:27.

10. Maheswari J., Mataliya MV. Diabetes in pregnancy. J Obstet Gynaecol India. 1989;39:351.

11. Lawrence JM, Conteras R, Chen W, Sacks DA. Trends in the prevalence of pre-existing diabetes and gestational diabetes mellitus among a racially/ ethnically diverse population of pregnant women. 1999-2005. Diabetes Care. 2008;31(5):899-904.

12. Wahi P, Dogra V, Jandial K, Bhagat R, Gupta R, Gupta $S$, et al. Prevalence of gestational diabetes mellitus and its outcomes in Jammu region. J Assoc Physicians India. 2011;59(4):227-30. 
13. Rajput R, Yadav Y, Nanda S, Rajput M. Prevalence of gestational diabetes mellitus and associated risk factors at a tertiary care hospital in Haryana. Indian J Med Res.2013;137(4):728-33.

14. K Sreekanthan A, Belicit A, K Rajendran, Anil, Vijay Kumar. Prevalence of Gestational Diabetes Meliitus in a Medical College in South India: Pilot Study. Indian J Clinic Res. 2014;25(4):342-347.

15. Landon MB, Spong CY, Thom E, Carpenter MW, Ramin SM, Casey B, et al. A multicenter, randomized trial of treatment of mild gestational diabetes. New Eng J Med. 2009;361(14):1339-1348.

16. HAPO study cooperative research group. Hyperglycemia and adverse pregnancy outcome. New Eng J Med. 2008;358(19):1991-2002.

17. Cosson E. Screening and insulin sensitivity in gestational diabetes. In Abstract volume of the 40th Annual Meeting of the EASD 2004 Sep (p. A350).

18. Getahun D, Nath C, Ananth CV, Chavez MR, Smulian JC. Gestational diabetes in the United States: temporal trends 1989 throught 2004. Am J Obstet Gynecol 2008;198(5): 525-e1.

19. Coustan DR, Lowe LP, Metzger BE. The hyperglycemia and adverse pregnancy outcome (HAPO) study: can we use the results as a basis for change? J Matern Fetal Neonatal Med 2010:23(3): 204-9.

20. Metzger BE, Gabbe SG, Persson B et al. International association of diabetes and pregnancy study groups recommendations on the diagnosis and classification of hyperglycemia in pregnancy. Diabtes Care. 2010;33(3):676-682.

21. Karcaaltincaba D, Kandemir O, Yalvac S, Güvendag-Guven S, Haberal A. Prevalence of gestational diabetes mellitus and gestational impaired glucose tolerance in pregnant women evaluated by National Diabetes Data Group and Carpeneter and Coustan criteria. Int J Gynaecol Obstet 2009;106(3): 246-9.

22. Bhavadharini B, Mahalakshmi MM, Anjana RM, Maheswari K, Uma R, Deepa M, et al. Prevalence of gestational diabetes mellitus in urban and rural Tamil Nadu using IADPSG and WHO 1999 criteria (WINGS 6). Clinic Diab Endocrinol. (2016);2(1):8.

23. O'Sullivan EP, Avalos G, O'reilly M, Dennedy MC, Gaffney G, Dunne F. Atlantic Diabetes in Pregnancy
(DIP): the prevalence and outcomes of gestational diabetes mellitus using new diagnostic criteria. Diabetol. 2011;54(7):1670-5.

24. Gopalakrishnan V, Singh R, Pradeep Y et al. Evaluation of the prevalence of gestational diabetes mellitus in North Indians using the International Association of diabetes and pregnancy study groups (IADPSG) criteria. J Postgrad Med. 2015;61(3):1558.

25. Hung TH. The effects of implementing the International Association of Diabetes and Pregnancy Study Groups criteria for diagnosing gestational diabetes on maternal and neonatal outcomes. PLoS One. 2015;10(3):e0122261.

26. Arora G, Thaman RG, Prasad R, Almgren P, Brøns $\mathrm{C}$, Groop L, et al. Prevalence and risk factors of gestational diabetes in Punjab, North India: results from a population screening program. Eur $\mathbf{J}$ Endocrinol.2015;173(2):257-67.

27. Nayak PK, Mitra S, Sahoo JP, Daniel M, Mathew A, Padma A. Fetomaternal outcomes in women with and without gestational diabetes mellitus according to the International association of diabetes and pregnancy study groups (IADPSG) diagnostic criteria. Diabe Metabol Syndrome: Clinical Res and Reviews. 2013;7(4):206-9.

28. Dahanayaka NJ, Agampodi SB, Ranasinghe OR, Jayaweera PM, Wickramasinghe WA, Adhikari AN et al. Inadequacy of the risk factor-based approach to detect gestational diabetes mellitus. Ceylon Med J. 2012;57(1):5-9.

29. Moradi S, Shafieepour MR, Motazavi M, Pishgar F. Prevalence of gestational diabetes in Rajasthan: a comparison of different criteria. Med J Islam Repub Iran. 2015;29:209.

30. Shang M, Lin L. IADPSG criteria for diagnosing gestational diabetes mellitus and predicting adverse pregnancy outcomes. J Perinatol. 2014;34(2):100-4.

Cite this article as: Chaudhary G, Raghunandan C, Chopra K. Screening and diagnosis of gestational diabetes mellitus by IADPSG criteria. Int J Reprod Contracept Obstet Gynecol 2019;8:543-7. 